\title{
Downregulation of matrix metalloproteinase-2 (MMP-2) utilizing adenovirus-mediated transfer of small interfering RNA (siRNA) in a novel spinal metastatic melanoma model
}

\author{
ANDREW J. TSUNG ${ }^{1}$, ODYSSEAS KARGIOTIS ${ }^{2}$, CHANDRAMU CHETTY ${ }^{2}$, SAJANI S. LAKKA ${ }^{2}$, \\ MEENA GUJRATI $^{3}$, DANIEL G. SPOMAR ${ }^{1}$, DZUNG H. DINH ${ }^{1}$ and JASTI S. RAO ${ }^{1,2}$ \\ Departments of ${ }^{1}$ Neurosurgery, ${ }^{2}$ Cancer Biology and Pharmacology, and ${ }^{3}$ Pathology, \\ University of Illinois College of Medicine, Peoria, IL 61605, USA
}

Received September 11, 2007; Accepted November 8, 2007

\begin{abstract}
Matrix metalloproteinases (MMPs) comprise a class of secreted zinc-dependent endopeptidases implicated in the metastatic potential of tumor cells due to their ability to degrade the extracellular matrix (ECM) and basement membrane. Matrix metalloproteinase-2 (MMP-2) has been detected in high levels and correlates with invasiveness in human melanoma. We have studied the effect of adenovirusmediated transfer of small interfering RNA (siRNA) against MMP-2 in the human melanoma cell line A2058. The delivery of these double-stranded RNA molecules represents an efficient technology in silencing disease-causing genes with known sequences at the post-transcriptional level. siRNA against MMP-2 mRNA (Ad-MMP-2) was found to decrease MMP-2 protein expression and activity in melanoma cells as demonstrated by Western blotting and gelatin zymography. Furthermore, infection of cells with Ad-MMP-2 inhibited cellular migration and invasion as indicated by spheroid and matrigel assays. We also observed dosedependent suppression of vascular network formation in an angiogenesis assay. Finally, we developed a nude mouse spinal metastatic model to investigate the local effects of tumor metastasis. Intravenous tail vein injection with AdMMP-2 on days 5,9 and 11 after tumor implantation resulted in complete retention of neurological function as
\end{abstract}

Correspondence to: Dr Jasti S. Rao, Department of Cancer Biology and Pharmacology, University of Illinois College of Medicine at Peoria, One Illini Drive, Peoria, IL 61605, USA

E-mail: jsrao@uic.edu

Abbreviations: MMP, matrix metalloproteinase; Ad-MMP-2, adenoviral construct for MMP-2 siRNA; Ad-SV, adenoviral construct for scrambled sequence construct for control; TIMP, tissue inhibitor of metalloproteinase; siRNA, small interfering RNA; ECM, extracellular matrix

Key words: MMP-2, melanoma, invasion, spheroid migration, angiogenesis, metastatic model compared to control and scrambled vector (Ad-SV)-treated groups that showed complete paraplegia by day $14 \pm 2$ days. Hematoxylin and eosin staining revealed decreased tumor size in the Ad-MMP-2-treated animals. This novel experimental model revealed that adenoviral-mediated transfer of RNA interference against MMP-2 results in the retention of neurological function and significantly inhibited tumor growth.

\section{Introduction}

Although cutaneous melanoma represents only $1 \%$ of all cancers and accounts for approximately 50,000 new cases per year, this melanoma has one of the most rapidly increasing incidences of all solid tumors $(1,2)$. It is one of the most common and aggressive cancers, occurring in the third decade of life and often presenting with extensive skeletal metastasis at the time of diagnosis $(3,4)$. Although vertebral metastasis causes a diminished quality of life as a result of pain and paraplegia, treatment options remain undefined and ineffective (5). Complicating matters further is the fact that melanoma is a radioresistant tumor, leaving even less options available to the treating physician (6). Thus, effective pharmacologic alternatives to halt the progression of disease are needed to augment the insufficient medical and surgical armamentarium, as it currently exists.

As a lesion originating within the epidermis, malignant melanomas possess unique characteristics for efficient dissemination throughout the body. Among these is the group of zinc-dependent endopeptidases termed matrix metalloproteinases (MMPs). These enzymes have wide-ranging roles in non-pathological and pathological states as they are involved in a variety of tissue remodeling processes including wound healing, cellular migration, invasion and angiogenesis (7-9). More than 20 molecules belong to this family and are subdivided into collagenases, gelatinases, stromelysins, matrilysins and membrane-type MMPs. In vitro data have specifically implicated the $72-\mathrm{kD}$ gelatinase MMP-2 in the pivotal role of melanoma invasion. MMP-2 is secreted as a latent pro-form and processed into an active enzyme through cell surface interactions with specific tissue inhibitors (TIMPs) (10). Of all the MMPs and TIMPs assayed in a 
human melanoma xenograft model, only the expression of activated MMP-2 correlated with the aggressive malignant phenotype (11). Other studies have duplicated this observation in other models (12-15). Clinical aspects of MMP-2 expression have been validated in human biopsy specimens where a $70.5 \%$ negative rate of immunohistochemical expression was found in patients with good prognosis compared with only a $47 \%$ negative rate in those who developed metastasis (16). Serum levels of MMP-2 have also been found to be elevated in those with metastatic melanoma compared to those with unifocal disease. However, at this point, the clinical application of this information remains undefined $(16,17)$.

In the present study, we used RNA interference (RNAi) to downregulate the expression of MMP-2. RNAi, and more specifically, small interfering RNA (siRNA), has emerged as a potent tool in post-transcriptional regulation due to its inherent specificity for known gene sequences and its versatility against many pathogens as diverse siRNAs can be designed (18). In the present study, we utilized an adenovirus-mediated method of transferring siRNA as recent studies have shown this method to be a more efficient delivery mechanism $(19,20)$. Our results confirm that adenovirus-mediated transfer of siRNA against MMP-2 resulted in downregulation with subsequent decreases in invasion, migration and angiogenesis. Furthermore, for the first time, we describe a novel metastatic spine model in nude mice in which we validate these results via $100 \%$ retention of neurological function and decreased tumor growth as compared to control animals.

\section{Materials and methods}

Cell cultures and adenoviral constructs. The human melanoma cell line A2058 was grown in Dulbecco's modified Eagle's medium (DMEM). Cultures were supplemented with $1 \%$ glutamine, $100 \mu \mathrm{g} / \mathrm{ml}$ streptomycin, $100 \mathrm{U} / \mathrm{ml}$ penicillin, and $10 \%$ fetal bovine serum (pH 7.2 to 7.4 ) and maintained in a humidified atmosphere containing $5 \% \mathrm{CO}_{2}$ at $37^{\circ} \mathrm{C}$. The adenoviral constructs carrying siRNA against MMP-2 (Ad-MMP-2) and a scrambled MMP-2 sequence (Ad-SV) were designed and constructed as described previously (20). Briefly, the following siRNA sequences were cloned into the pSuppressor vector: 5'-AACGGACAAAGAGTTG GCAGTATCGATACTGCCAACTCTTTGTCCGTT-3' for Ad-MMP-2; and 5'-GCACGGAGGTTGCAAAGAATAA TCGATTATTCTTTGCAACCTCCGTGC-3' for Ad-SV. We used the adenoviral pSuppressor kit (Imgenex, San Diego, CA) to create the final constructs as per the manufacturer's instructions. The pSuppressor plasmids were digested with PacI and co-transfected with pAd vector backbone in 293 cells for recombinant generation of the adenoviruses carrying MMP-2 siRNA and the scrambled sequence. Viruses were plaque purified and used to infect 293 cells, and subsequently purified on a cesium chloride gradient and tittered as previously described (20). The amount of infective adenoviral vector per cell (pfu/cell) in culture media was expressed as multiplicity of infection (MOI). Virus constructs were diluted in serum-free culture media to the desired concentration and added to cells and incubated at $37^{\circ} \mathrm{C}$ for $1 \mathrm{~h}$.
The necessary amount of complete medium was then added and cells were incubated for the desired time periods.

Gelatin zymography. Cells were infected with mock, Ad-SV (100 MOI) or Ad-MMP-2 (12.5, 25, 50 and 100 MOI). Twenty-four hours after infection, conditioned medium was replaced with serum-free medium and cells were incubated overnight. Thirty micrograms of each sample were assayed for gelatinase activity as described previously (20). Briefly, electrophoresis was performed on $10 \%$ SDS-PAGE containing $1 \mathrm{mg} / \mathrm{ml}$ gelatin. Gels were washed in $2.5 \%$ Triton $\mathrm{X}-100$ and incubated overnight at $37^{\circ} \mathrm{C}$ in a buffer $(50 \mathrm{mmol} / 1$ Tris- $\mathrm{HCl}, 0.05 \% \mathrm{NaN}_{3}, 5 \mathrm{mmol} / 1 \mathrm{CaCl}_{2}$ and $1 \mu \mathrm{mol} / 1 \mathrm{ZnCl}_{2}$, $\mathrm{pH}$ 7.6). Gels were stained with Amido black solution, destained and MMP-2 activity was visualized as clear bands on a dark background.

Western blot analysis. A2058 cells were mock infected or infected with the indicated MOI of Ad-SV and Ad-MMP-2. Equal amounts of total protein from conditioned media or cell lysates obtained by lysing cells in a suitable buffer [50 mmol/l Tris-HCl (pH 8.0), 150 mmol/1 NaCl, $1 \%$ NP40, $0.5 \%$ sodium deoxycholate, $0.1 \% \mathrm{SDS}, 0.5 \mathrm{mmol} / 1$ phenylmethylsulfonylfluoride] were separated on SDS-PAGE and transferred to polyvinylidene difluoride membranes (Bio-Rad). After blocking with $6 \%$ non-fat milk and $0.1 \%$ Tween-20 in TBS, membranes were incubated with antiMMP-2 antibody (Chemicon, Temecula, CA), followed by incubation with anti-mouse secondary antibody. Membranes were developed using the ECL system (Amersham Bioscience, Piscataway, NJ). GAPDH antibody was used as a loading control.

Matrigel invasion assay. A2058 cells infected with mock or adenoviral constructs (100 MOI of Ad-SV; 50 and 100 MOI of Ad-MMP-2) were trypsinized and counted. Cells $\left(2 \times 10^{5}\right)$ were placed in matrigel-coated transwell inserts $(8-\mu \mathrm{m}$ pores). Cells were allowed to migrate through the matrigel for $48 \mathrm{~h}$. Cells were then removed from the upper chamber with a cotton swab. Cells adherent to the outer surface that had invaded through the matrigel were fixed, stained with HEMA and counted under a light microscope as described previously (21).

Spheroid migration assay. A2058 cells were seeded in 96-well plates coated with $1 \%$ agarose in PBS and cultured on a shaker at $90 \mathrm{rpm}$ for $48 \mathrm{~h}$. After single spheroids formed, cells were infected with mock, Ad-SV (100 MOI), or Ad-MMP-2 (25, 50 and 100 MOI). After $24 \mathrm{~h}$, spheroids were transferred to 8 -well chamber slides and allowed to grow for $48 \mathrm{~h}$. The results were observed under a confocal scanning laser microscope and the migration distance was measured using Image Pro Discovery software (21).

In vitro angiogenesis assay. Briefly, A2058 melanoma cells were infected with mock, Ad-SV (100 MOI), or Ad-MMP-2 (25, 50 and $100 \mathrm{MOI})$. After $36 \mathrm{~h}$, cells were washed with serum-free medium and incubated overnight. The conditioned media was removed and added to a monolayer of $2 \times 10^{4}$ human microvascular endothelial cells in 96-well plates 


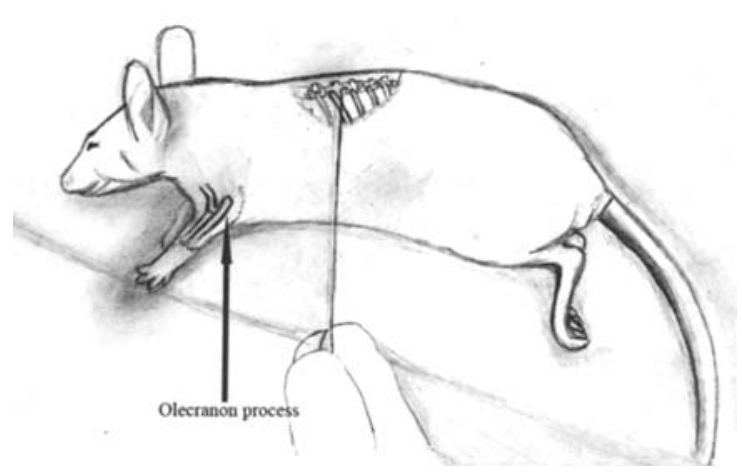

Figure 1. Novel nude mouse model for metastatic spinal melanoma. Eightto 12 -week-old nude mice were cared for according to guidelines and protocols established by the Institutional Animal Care and Use Committee (IACUC). Animals were positioned in either right or left lateral decubitus position after anesthesia induction. In this position, a natural line forms originating parallel to the ulna extending out from the olecranon process. This line intersects the spine at approximately the T10 vertebrae. A $1.5-\mathrm{cm}$ incision was made parallel to this line. The \#15 scalpel blade was used to dissect through superficial and deep fascia to the depth of the spine. A Hamilton syringe with a blunt tip was then 'walked' along the rib from distal to the proximal intersection with the vertebral body. Tactile feedback and moderate pressure assured that the syringe tip was within the vertebral body. All 3 groups of animals were then injected with $1 \times 10^{6}$ melanoma cells $/ \mu 1$ suspended in serum-free Dulbecco's modified Eagle's medium (DMEM) for $5 \mathrm{~min}$. The skin was closed with glue. Serum-free, Ad-SV, or Ad-MMP-2 ( $1 \times 10^{8}$ particles $)$ tail vein injections were then performed on days 5, 9 and 11. Animals were sacrificed on day 18 or after the onset of paraplegia, whichever came first.

coated with matrigel. Cells were allowed to grow overnight. Next, cells were washed with PBS and the formation of capillary-like structures was captured using light microscopy. The angiogenic result was measured by counting the relative branch points in each field.

Novel metastatic spine model. Due to the lack of an established, reproducible animal model that allows for easy analysis of tumor progression and resultant paralysis, a novel metastatic spine model was developed using 8- to 10week-old nude mice (Fig. 1). Animals were placed in either the right or left lateral decubitus position after anesthesia induction via intraperitoneal injection with ketamine $50 \mathrm{mg} /$ $\mathrm{kg}$ and xylazine $10 \mathrm{mg} / \mathrm{kg}$. In this position, a natural line forms that originates parallel to the ulna extending out from the olecranon process. This line intersects the spine at approximately the T10 vertebrae. A $1.5-\mathrm{cm}$ incision was made parallel to this line. A \#15 scalpel blade was used to dissect through superficial and deep fascia to the depth of the spine. A Hamilton syringe with a blunt tip was then 'walked' along the rib from distal to the proximal intersection with the vertebral body. The needle was then translated anteriorly approximately 1-2 mm to avoid the spinal cord and canal. Tactile feedback and moderate pressure assures the syringe tip is within the vertebral body. Animals were then injected with $1 \times 10^{6}$ melanoma cells $/ 5 \mu 1$ suspended in serum-free Dulbecco's modified Eagle's medium (DMEM) for $5 \mathrm{~min}$. The skin was closed with glue. Groups were divided into serum-free (control), Ad-SV, or Ad-MMP-2 (1 $\times 10^{8}$ particles/ $50 \mu 1$ ) treatment groups (5 animals per group) and tail vein injections were performed accordingly on days 5,9 and 11 .
A
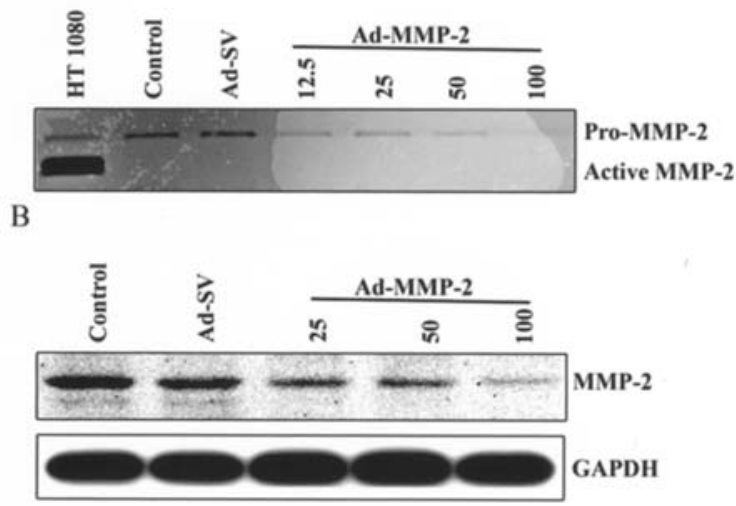

Figure 2. Adenoviral-mediated transfer of siRNA (Ad-MMP-2) downregulates MMP-2 protein activity and levels. The human melanoma cell line A2058 was infected with Ad-MMP-2 (12.5, 25, 50 and $100 \mathrm{MOI})$, scrambled vector (Ad-SV; $100 \mathrm{MOI}$ ), or mock (Control). After $72 \mathrm{~h}$, conditioned media were collected for gelatin zymography analysis (A) and equal amounts of total protein from the conditioned media were used for Western blot analysis of MMP-2 protein (B). GAPDH was used as a loading control.

Animals were monitored twice daily for the onset of paraplegia. Animals were sacrificed on day 18 or after the onset of paraplegia.

Immunohistochemistry. Spinal cord and columns were removed en bloc and stored in formalin, subsequently decalcified and embedded in paraffin. Hematoxylin and eosin (H\&E) was used to visualize cellular morphology, patterns of invasion and mass effect upon the spinal cord. For immunohistochemistry, spinal sections were heated to $65^{\circ} \mathrm{C}$ for $1 \mathrm{~h}$, deparaffinized in xylene and rehydrated through graded alcohol. Slides were incubated with $0.1 \%$ Triton X-100, blocked with 3\% BSA in PBS and incubated with antibodies against HMB45 and MART-1 (Abcam, Cambridge, MA), both of which are ubiquitous antigens found in melanoma. After a rinse in PBS, slides were exposed to secondary anti-mouse antibody for $1 \mathrm{~h}$ at a dilution of 1:200, washed again with PBS and incubated with $0.05 \%$ 3,3'-diaminobenzidine as chromogen. Finally, slides were counterstained with hematoxylin, mounted and observed under a light microscope.

\section{Results}

Ad-MMP-2 downregulates MMP-2 protein levels and enzyme activity. To verify that this construct efficiently downregulated the MMP-2 transcript, we infected A2058 human melanoma cells with 12.5, 25, 50, or $100 \mathrm{MOI}$ of Ad-MMP-2. These results were compared to $100 \mathrm{MOI}$ of $\mathrm{Ad}-\mathrm{SV}$ and the control (non-infected) group. Using conditioned media from the cultured cells, gelatin zymography analysis was performed to determine the activity of the MMP-2 secreted into the media. The $72-\mathrm{kDa}$ bands were progressively less intense with increasing MOI of Ad-MMP-2 demonstrating a dose-dependent MMP-2 inhibition compared to control and Ad-SV-treated cells (Fig. 2A). Equal amounts of protein were obtained from conditioned media of treated and 


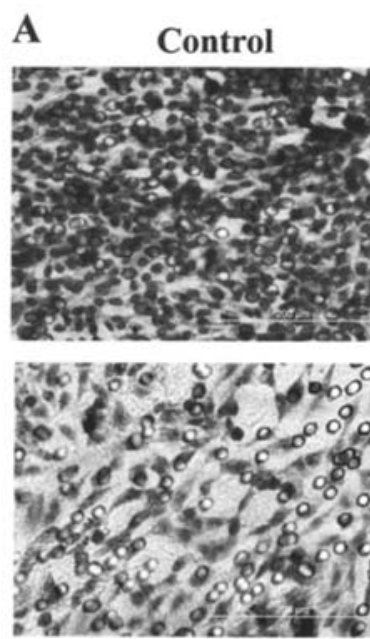

50 MOI
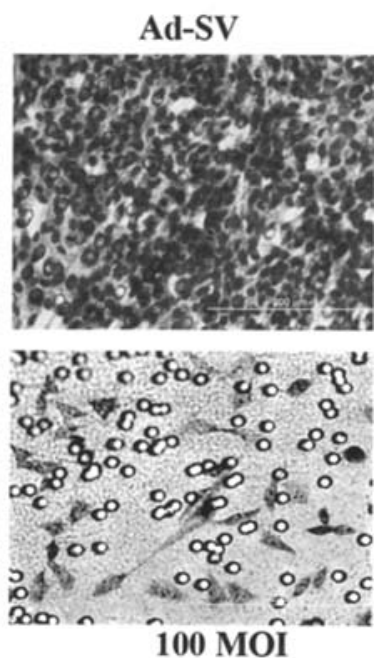

B

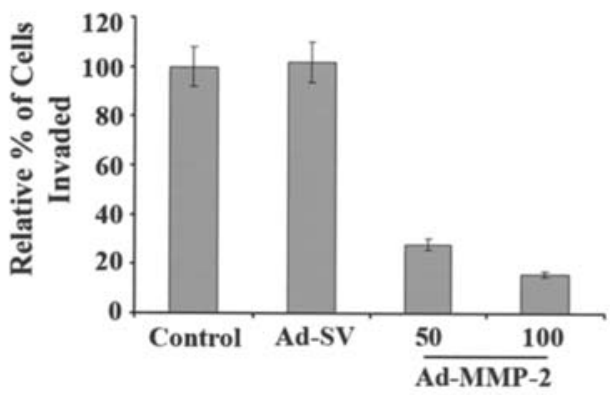

Figure 3. Downregulation of MMP-2 inhibits cell invasion. A2058 cells were infected with mock, Ad-SV (100 MOI), or Ad-MMP-2 (50 and 100 MOI). Twenty-four hours later, cells were trypsinized and $2 \times 10^{5}$ cells were placed in transwell chambers coated with matrigel at $0.9 \mathrm{mg} / \mathrm{ml}$. After $48 \mathrm{~h}$, cells were fixed, stained with HEMA and photographed (A). Quantification of invasion as percentage of control (B). Data shown are mean values of four different experiments from each group.

untreated groups for Western blot analysis and demonstrated significantly decreased protein levels of MMP-2 with AdMMP-2 constructs compared to Control and Ad-SV-treated cells (Fig. 2B). These results correspond with those found with gelatin zymography.

Ad-MMP-2 inhibits melanoma cell invasion. The extracellular matrix (ECM) is the largest obstacle in preventing metastasis of the melanoma cell. MMP-2 has been implicated in the digestion of the basement membrane, which is largely composed of Type IV collagen, also called gelatin. For this reason, we chose to investigate the effect of downregulating MMP-2 via siRNA-mediated methods. The matrigel invasion assay results showed dose-dependent inhibition of tumor cell invasion when treated with Ad-MMP-2 compared to control and Ad-SV-treated cells (Fig. 3A). After normalization of values as a percentage of the control (100\%), those with the highest MOIs of Ad-MMP-2 showed 80-85\% inhibition of tumor cell invasion (Fig. 3B).

Ad-MMP-2 completely inhibits tumor cell migration. Tumor migration is enhanced by MMP-2 as tumor cells degrade the ECM, releasing growth factors necessary for translation through the extracellular milieu. Additionally, there is significant binding to integrins, which serve as anchoring

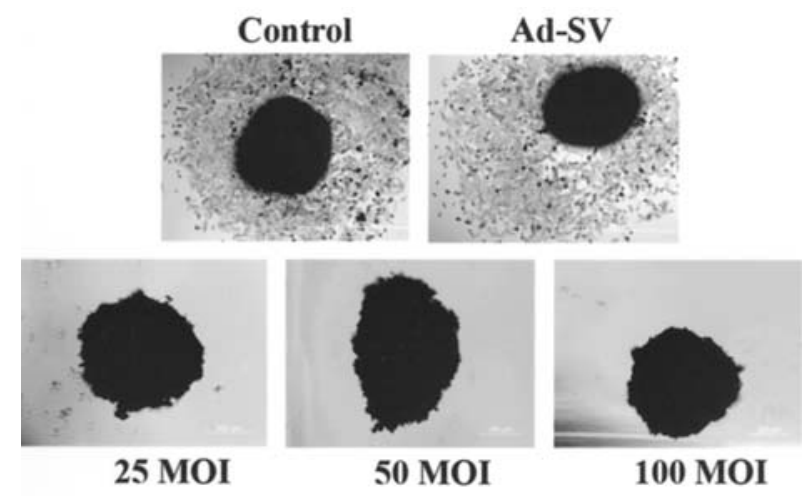

Figure 4. Ad-MMP-2 reduces tumor cell migration. A2058 tumor spheroids were seeded in 96-well plates coated with $1 \%$ agarose in PBS and cultured on a shaker at $90 \mathrm{rpm}$ for $48 \mathrm{~h}$. After spheroid formation, cells were infected in the following groups: mock, Ad-SV (100 MOI), or Ad-MMP-2 (25, 50, and $100 \mathrm{MOI}$ ). After $24 \mathrm{~h}$, spheroids were transferred to 8-well chamber slides, and allowed to grow for $72 \mathrm{~h}$. Cold methanol fixation was performed and followed by HEMA staining.

A

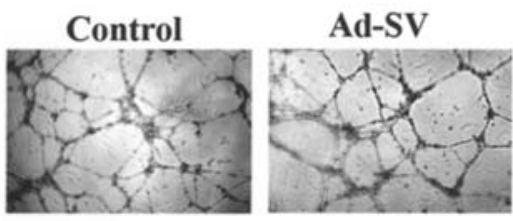

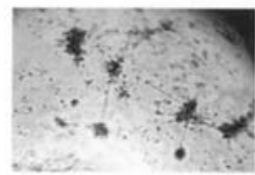

B

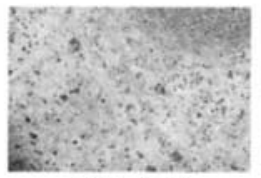

50 MOI

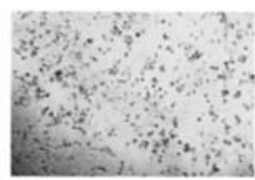

$100 \mathrm{MOI}$

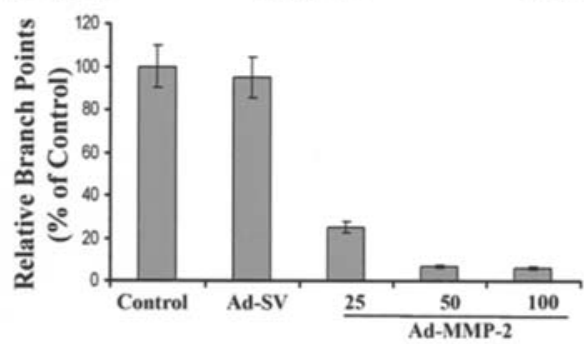

Figure 5. In vitro inhibition of angiogenesis. Human microvascular endothelial cells (HMECs) were plated at $2 \times 10^{4}$ onto diluted matrigel tissue culture plates and grown in the presence of media from A2058 control cells, or A2058 cells treated with either Ad-SV (100 MOI) or Ad-MMP-2 (25, 50 and $100 \mathrm{MOI}$ ) for $12 \mathrm{~h}$ or overnight (A). Branch points were calculated using computer-assisted image analysis with the Image-Pro software and tabulated as a percentage of the control (B). Data shown are mean values of four different experiments from each group.

points on the advancing tumor front. Because tumor spheroids mimic tumor migratory characteristics, we formed A2058 spheroids and infected them with 25, 50, or $100 \mathrm{MOI}$ of Ad-MMP-2 and compared these results to the control and Ad-SV groups. Our results show a significant radial migration pattern in the control and Ad-SV groups as compared to complete inhibition at even the lowest doses of Ad-MMP-2 (Fig. 4).

Ad-MMP-2 reduces angiogenesis in vitro. Metastatic tumor cells cannot exist beyond an approximate distance of $1 \mathrm{~mm}$ 

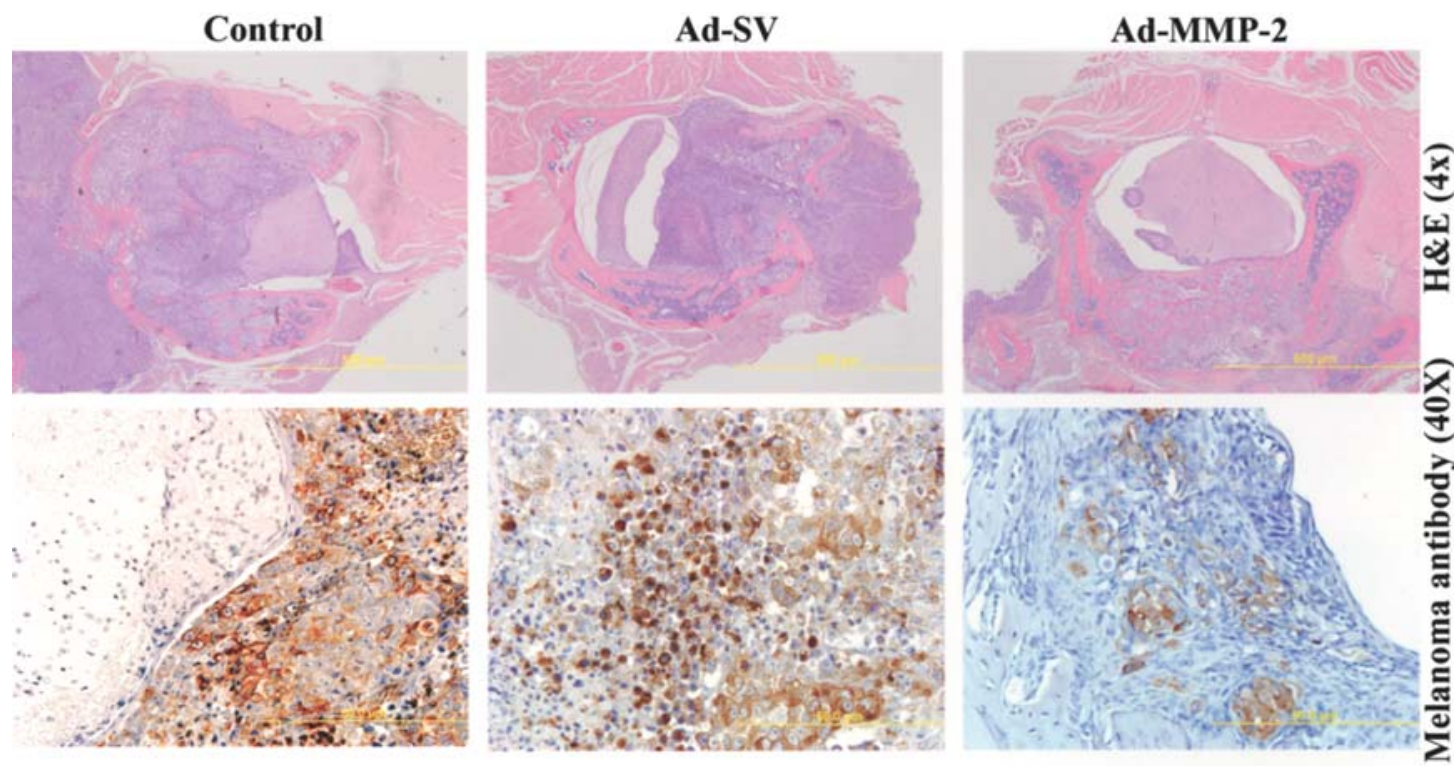

Figure 6. Tumor growth inhibition in nude mice. Melanoma cells $\left(1 \times 10^{6}\right)$ were injected as described in Fig. 1. On days 5 , 9 and 11 , serum-free media (control), Ad-SV or Ad-MMP-2 were injected through tail veins. Tumor sections were stained with hematoxylin and eosin (H\&E). Immunohistochemical analysis for melanoma antibody [HMB45+DT101+BC199], targeting the cytoplasmic proteins HMB45 and MART-1.

from a direct vascular source, thus requiring angiogenic factors for the continued existence and subsequent growth of tumor cell deposits. Numerous studies have established that MMPs have significant roles in inducing angiogenesis. Here, our results demonstrate that Ad-MMP-2-treated melanoma cells have significantly decreased capillary-like formation in a dose-dependent fashion (Fig. 5A). At the highest MOI of Ad-MMP-2, we observed an almost complete repression of the vascular network (Fig. 5B) compared to control and Ad-SV-treated cells.

Ad-MMP-2 treatment allows for complete retention of neurological function in vivo. We developed a nude mouse model to study neoplasia, and specifically metastasis of the bony spine. All the control and Ad-SV-treated mice exhibited consistent paraplegia by day $14 \pm 2$ days with an injection density of $1 \times 10^{6}$ melanoma cells $/ 5 \mu 1$. The paraplegia was abrupt in nature without any preceding gradual loss of function. Notably, the Ad-MMP-2-treated group showed no evidence of paraplegia or diminished neurological function for the duration of the experiment. Furthermore, there was no evidence of morbidity from the surgical procedure, weight loss, or systemic side effects from treatment.

Ad-MMP-2 diminishes tumor size and minimizes mass effect on the spinal cord. After performing immunohistochemistry on the tissue sections, we observed clear visualization of cellular morphology and invasion characteristics of human melanoma. In the control and Ad-SV groups, H\&E staining showed sheets of pleomorphic cells with large nucleoli invading from an anterior and anterolateral direction in relation to the spinal cord, mimicking the human pathophysiological state. Furthermore, these cells were found to have proliferated to an extensive degree, which in turn, caused significant compression that often times resulted in distorted spinal cord morphology resembling a crescent (Fig. 6). Melanoma antibody analysis showed that despite proliferation, migration and invasion, there was no subsequent invasion of the neural elements, including the spinal cord. This indicates that the paraplegia was caused solely by physical compression rather than cellular invasion of the neuraxis.

In contrast, the Ad-MMP-2-treated group had significant reduction in tumor size (Fig. 6). These results are in accord with the functional results, as all Ad-MMP-2-treated animals retained the ability to walk. Immunohistochemistry did reveal tumor deposits present in the same locations as the control and Ad-SV groups. However, the deposits were diminished in size with no signs of mass effect upon the spinal cord or other neural elements. Thus, while treatment was given intravenously rather than in situ due to the impossibility of that mode of treatment in this model, we were still able to demonstrate in vivo efficacy of systemic adenoviral vector delivery of siRNA.

\section{Discussion}

The current study demonstrates the in vitro and in vivo efficacy of adenovirus-mediated transfer of siRNA against the MMP-2 gene in a novel spinal metastatic melanoma model. The use of adenoviral vectors for gene therapy in cancer is the delivery method of choice due to high infectivity and lack of integration into the host genome, which limits the risk of insertional mutagenesis. Additionally, expression of the adenoviral genome is of short duration without integration, which is all that is necessary for tumor therapy (as compared with chronic genetic disorders that require long-term expression) (22). From this perspective, our results substantiate previous claims as infectivity and expression were high as shown by the in vitro results, lack of mutagenic effects, and retention of neurological function in all treated animals. Furthermore, few studies exist which have used systemic (intravenous) delivery of adenoviral siRNA sequences rather than local intratumoral injection $(20,23)$. 
Our results demonstrate that it is possible to treat neoplasia to the degree of preventing neurological deficit via the intravenous delivery method, and illustrate the potential of systemic adenoviral delivery of siRNA. This novel and reproducible animal model that we developed to study metastatic spinal melanoma can be applied to practically any tumor and allows for the observation of physical behavior correlating with tumor growth.

RNAi has already been demonstrated as a potent therapy for targeting a wide variety of diseases (24-26). Much of the potential of RNAi is derived from a considerable posttranscriptional silencing effect even at a low concentration; the concentrations required are usually several magnitudes lower than that necessary for antisense oligonucleotide therapy $(27,28)$. Although not integrated into the genome, RNAi activity has been shown to last for more than three weeks via the stability afforded by the RNA interfering silencing complex (RISC) (29). Phase I and II human trials are already underway to determine the safety and effectiveness of local delivery of siRNA for treatment of respiratory synctial virus (RSV) and macular degeneration, and future studies for the treatment of hepatitis, amyotrophic lateral sclerosis (ALS) and HIV are anticipated $(18,30)$.

Cancer cell invasion and metastasis are complex, multifaceted and multi-step processes, requiring the interaction of cancer cells with the host environment. Within this milieu, there exist many enzymes necessary for the controlled digestion of the basement membrane and extracellular domain. This includes the plasminogen activator system, aspartyl proteinases, cysteine proteinases, serine proteinases and matrix metalloproteinases. Of these enzymes, general inhibition of MMPs has shown a benefit in melanoma. For example, the synthetic non-specific hydroxamate-type MMP inhibitor batimastat had a $68 \%$ inhibition of lung tumor deposits in a B16 murine metastatic melanoma model (31). The support for MMP-2 as a pivotal enzyme in metastasis is strong as this zinc-dependent gelatinase (also known as 72-kDa type IV collagenase) is highly expressed in malignant phenotypes characterized by increasing architectural disorder, atypia and progression (11,32,33). MMP-2-deficient mice also showed reduced angiogenesis and tumor progression in a metastatic model (34). Furthermore, the data support a correlation between MMP-2 expression and the prognosis of a melanoma patient (35). Because MMP-2 is upregulated in the vertical growth phase of melanoma, and thus, may represent a state of increased invasion and proliferation potential, we hypothesized that specifically targeting MMP-2 would result in significant growth inhibition.

The adenoviral delivery of siRNA against MMP-2 has already been described in the treatment of cancer. For example, in a study involving lung cancer metastasis, siRNA against MMP-2 resulted in decreased invasion, migration and angiogenesis with a resultant $60 \%$ reduction in tumor volume in treated animals (20). Other methods of downregulation have validated MMP-2 as a practical target. Montgomery et al showed that cDNA transfection with tissue inhibitor of metalloproteinase-2 (TIMP-2) markedly reduced melanoma growth in a cutaneous immunodeficient mouse model although metastasis still ensued (36). The reasons for the observed growth inhibition seen with MMP-2 down- regulation are likely multi-factorial. In the initial phase of metastasis, invasion and migration are key steps. In the present study, the matrigel assay results show that MMP-2 downregulation resulted in an $>80 \%$ inhibition of invasion as compared to the control. This method mimics the basement membrane barrier to invasion, thus illustrating the interaction between tumor cells and the microenvironment. Melanoma cells utilize precise methods of digestion occurring in conjunction with simultaneous adhesion to the stroma. It has been demonstrated that at the invasion front of melanoma, this is characterized by upregulation of MMP- 2 only in the aggressive cell lines while MMP-9, TIMP-1 and MT1-MMP were expressed in all cells at the tumor-stromal interface (37). This illustrates the specific actions of MMP-2 not only in the context of its function, but also in its localization of function during metastatic tumor formation.

Post-transcriptional gene control via intravenous injection of adenovirus is classified as non-specific, meaning that theoretically, the DNA sequence is delivered to all cells without tropism towards a specific tissue type other than the known adenoviral affinity for the liver. We propose that some of the growth inhibition observed in vivo may have resulted from the intravenous delivery mechanism, as stromal cells of the bone and peri-spinal region are also theoretically exposed to siRNA. Others have already shown that cells other than the tumor itself can be the major source of MMP-2 in melanoma (38) and tumor growth factors such as the extracellular matrix metalloproteinase inducer (EMMPRIN; CD 147) can induce surrounding fibroblasts to secrete MMP-2 $(39,40)$. In addition, the issue of tumor versus stroma as the source of MMPs is highly variable depending on the tissue studied (37). This observation perhaps strengthens the argument for systemic delivery as one would not need to deliberate the efficacy between intratumoral and intravenous therapy.

Once tumor cells have spread through the vasculature and seeded elsewhere, angiogenesis is a necessary component for subsequent tumor growth as malignant cells with a volume of $>1-2 \mathrm{~mm}^{3}$ exceed the maximum diffusability of required nutrients necessary to sustain tumor growth (41). Thus, in the absence of neovascularization, solid tumor deposits could not exceed a certain, small dimension. Our results support this mechanism as the immunohistochemical data show the presence of tumor in the treated animals but failure of sustained growth. This may be due to the downregulation of MMP-2-induced angiogenesis. This assertion is corroborated by our in vitro data, which demonstrate the vascular tubular network was repressed to $30 \%$ of control levels. Indeed, others have shown this same effect. Fang et al demonstrated via gel electrophoresis that the switch to the angiogenic phenotype correlated with an increase in MMP-2 in a rat chondrosarcoma model (42). Specific inhibition of MMP-2 resulted in decreased angiogenic and proteolytic activity of tumor nodules and suppressed in vivo tumor growth by $70 \%$. The vitronectin-binding integrin $\alpha_{v} \beta_{3}$, which is highly expressed in melanoma metastases, binds active MMP-2 on the cell surface of endothelial cells and apparently promotes vascular proliferation. Active blocking of this interaction results in reduced tumor vascularity and size (43). Radiationinduced angiogenesis was also found to be partially mediated 
by MMP-2 in B16 melanoma cells. This effect was abrogated by the MMP inhibitor metastat (44).

Our rationale for developing a novel model was the lack of established protocols in studying neoplasia and the spine. A previous model utilizes left ventricular intracardiac injection in a murine metastatic B16 melanoma model (45). While the yield was high with tumor nodules forming in $100 \%$ of animals, there was widespread metastatic involvement from the onset. With this high tumor load, the animal may deteriorate physically which may limit survival, thus affecting final analysis of the treatment results. Furthermore, this model may significantly alter or lower the volume of distribution in systemically administered adenovirus as other studies have found clustering of siRNA in and around the tumor neovasculature (22). More recent animal models describe anatomically correct but technically difficult procedures in rabbits, requiring drilling and surgical expertise (46). In contrast, our model requires little training and materials, and isolates metastatic disease to the spine with physically observable effects of tumor progression, namely paraplegia. Furthermore, we sought to recreate the pathophysiological state of spinal cord compression from metastatic tumor involvement of the vertebral body. In humans, the vast majority of tumor deposits occur in the vertebral body due to the presence of large amounts of hematopoietic marrow (47). Resultant growth and local spread of the tumor results in spinal cord compression from an anterior and lateral direction (48). As evidenced by the immunohistochemistry results, we were able to successfully mimic this scenario. Our results also indicate a correlation between paraplegia and the amount of tumor burden. Those with intact function had little tumor deposit present within the vertebral body while those with paraplegia had wide-spread tumor involvement from the anterior and lateral directions with direct compressive forces resulting in significant distortion of the spinal cord.

The present study validates systemic adenoviral siRNA delivery and the targeting of MMP-2 in metastatic melanoma. We have demonstrated significantly diminished tumor cell invasion, migration and angiogenesis. In vivo, these results correlated with retention of neurological function in a novel metastatic spinal model. While other metastatic models to date rely upon either intracardiac injection or technically more involved procedures, this model relies upon simple anatomical concepts with reproducible results and confirmed immunohistochemical mimicry of the human pathophysiological state. The fact that tumor was still observed within the vertebrae of treated animals imply that the RNAi approach, while valid, may require a multimodal strategy aimed at all the specific steps involved in metastasis. With foreseeable improvements in siRNA delivery and specificity, proteases such as MMP-2 may become attractive targets in the clinical therapy of cancer and metastatic disease.

\section{Acknowledgements}

The authors thank Shellee Abraham for preparing the manuscript and Diana Meister and Sushma Jasti for the manuscript review. We thank Noorjehan Ali for technical assistance. We also thank Michael Copp for his assistance with immunohistochemistry and Andreana Maroon for illustrations. This research was supported by National Cancer Institute Grants CA 75557, CA 92393, CA 95058, CA 116708, N.I.N.D.S. NS47699 and NS057529 and Caterpillar, Inc., OSF Saint Francis, Inc., Peoria, IL (to J.S.R.).

\section{References}

1. Douglas JG and Margolin K: The treatment of brain metastases from malignant melanoma. Semin Oncol 29: 518-524, 2002.

2. Gerszten PC, Burton SA, Quinn AE, Agarwala SS and Kirkwood JM: Radiosurgery for the treatment of spinal melanoma metastases. Stereotact Funct Neurosurg 83: 213-221, 2005.

3. Gokaslan ZL, Aladag MA and Ellerhorst JA: Melanoma metastatic to the spine: a review of 133 cases. Melanoma Res 10: $78-80,2000$

4. Spiegel DA, Sampson JH, Richardson WJ, Friedman AH, Rossitch E, Hardaker WT Jr and Seigler HF: Metastatic melanoma to the spine. Demographics, risk factors and prognosis in 114 patients. Spine 20: 2141-2146, 1995.

5. Patchell RA, Tibbs PA, Regine WF, Payne R, Saris S, Kryscio RJ, Mohiuddin M and Young B: Direct decompressive surgical resection in the treatment of spinal cord compression caused by metastatic cancer: a randomised trial. Lancet 366 : 643-648, 2005.

6. Overgaard $\mathrm{J}$ : The role of radiotherapy in recurrent and metastatic malignant melanoma: a clinical radiobiological study. Int J Radiat Oncol Biol Phys 12: 867-872, 1986.

7. Chambers AF and Matrisian LM: Changing views of the role of matrix metalloproteinases in metastasis. J Natl Cancer Inst 89: 1260-1270, 1997.

8. Lakka SS and Rao JS: Role and regulation of matrix metalloproteases in brain tumors. In: Matrix Metalloproteinases in the Central Nervous System. Conant K and Gottschall PE (eds). Imperial College Press, London, pp263-278, 2005.

9. Ray JM and Stetler-Stevenson WG: The role of matrix metalloproteases and their inhibitors in tumour invasion, metastasis and angiogenesis. Eur Respir J 7: 2062-2072, 1994.

10. Hofmann UB, Westphal JR, van Muijen GN and Ruiter DJ: Matrix metalloproteinases in human melanoma. J Invest Dermatol 115: 337-344, 2000.

11. Hofmann UB, Westphal JR, Waas ET, Zendman AJ, Cornelissen IM, Ruiter DJ and van Muijen GN: Matrix metalloproteinases in human melanoma cell lines and xenografts: increased expression of activated matrix metalloproteinase-2 (MMP-2) correlates with melanoma progression. Br J Cancer 81: 774-782, 1999.

12. Capon F, Emonard H, Hornebeck W, Maquart FX and Bernard P: Expression and activation of pro-gelatinase A by human melanoma cell lines with different tumorigenic potential. Clin Exp Metastasis 17: 463-469, 1999.

13. Ikeda T, Murakami K, Sakukaw R, Hayakawa Y and Saiki I: Characterization of a liver metastatic variant of murine K1735M2 melanoma cells. In Vivo 14: 519-527, 2000.

14. Montgomery AM, De Clerck YA, Langley KE, Reisfeld RA and Mueller BM: Melanoma-mediated dissolution of extracellular matrix: contribution of urokinase-dependent and metalloproteinase-dependent proteolytic pathways. Cancer Res 53: 693-700, 1993.

15. Nakahara H, Howard L, Thompson EW, Sato H, Seiki M, Yeh Y and Chen WT: Transmembrane/cytoplasmic domainmediated membrane type 1-matrix metalloprotease docking to invadopodia is required for cell invasion. Proc Natl Acad Sci USA 94: 7959-7964, 1997.

16. Redondo P, Lloret $\mathrm{P}$, Idoate $\mathrm{M}$ and Inoges $\mathrm{S}$ : Expression and serum levels of MMP-2 and MMP-9 during human melanoma progression. Clin Exp Dermatol 30: 541-545, 2005.

17. Wollina U, Hipler UC, Knoll B, Graefe T, Kaatz M and Kirsch K: Serum matrix metalloproteinase-2 in patients with malignant melanoma. J Cancer Res Clin Oncol 127: 631-635, 2001 .

18. Kim DH and Rossi JJ: Strategies for silencing human disease using RNA interference. Nat Rev Genet 8: 173-184, 2007.

19. Brummelkamp TR, Bernards R and Agami R: Stable suppression of tumorigenicity by virus-mediated RNA interference. Cancer Cell 2: 243-247, 2002. 
20. Chetty C, Bhoopathi P, Joseph P, Chittivelu S, Rao JS and Lakka SS: Adenovirus-mediated siRNA against MMP-2 suppresses tumor growth and lung metastasis in mice. Mol Cancer Ther 5: 2289-2299, 2006.

21. Lakka SS, Rajan M, Gondi CS, Yanamandra N, Chandrasekar N, Jasti SL, Adachi Y, Siddique K, Gujrati M, Olivero W, Dinh DH, Kouraklis G, Kyritsis AP and Rao JS: Adenovirus-mediated expression of antisense MMP-9 in glioma cells inhibits tumor growth and invasion. Oncogene 21: 8011-8019, 2002.

22. Li CX, Parker A, Menocal E, Xiang S, Borodyansky L and Fruehauf JH: Delivery of RNA interference. Cell Cycle 5: 2103-2109, 2006.

23. Schmitz V, Vilanueva H, Raskopf E, Hilbert T, Barajas M, Dzienisowicz C, Gorschluter M, Strehl J, Rabe C, Sauerbruch T, Prieto J, Caselmann WH and Qian C: Increased VEGF levels induced by anti-VEGF treatment are independent of tumor burden in colorectal carcinomas in mice. Gene Ther 13: 1198-1205, 2006.

24. Duxbury MS, Matros E, Ito H, Zinner MJ, Ashley SW and Whang EE: Systemic siRNA-mediated gene silencing: a new approach to targeted therapy of cancer. Ann Surg 240: 667-674, 2004.

25. Filleur S, Courtin A, It-Si-Ali S, Guglielmi J, Merle C, Harel-Bellan A, Clezardin P and Cabon F: SiRNA-mediated inhibition of vascular endothelial growth factor severely limits tumor resistance to antiangiogenic thrombospondin-1 and slows tumor vascularization and growth. Cancer Res 63: 3919-3922, 2003.

26. Song E, Lee SK, Wang J, Ince N, Ouyang N, Min J, Chen J, Shankar P and Lieberman J: RNA interference targeting Fas protects mice from fulminant hepatitis. Nat Med 9: 347-351, 2003.

27. Coma S, Noe V, Lavarino C, Adan J, Rivas M, Lopez-Matas M, Pagan R, Mitjans F, Vilaro S, Piulats J and Ciudad CJ: Use of siRNAs and antisense oligonucleotides against survivin RNA to inhibit steps leading to tumor angiogenesis. Oligonucleotides 14: 100-113, 2004

28. Elbashir SM, Harborth J, Lendeckel W, Yalcin A, Weber K and Tuschl T: Duplexes of 21-nucleotide RNAs mediate RNA interference in cultured mammalian cells. Nature 411: 494-498, 2001.

29. Novina CD and Sharp PA: The RNAi revolution. Nature 430: 161-164, 2004.

30. Zhang W, Yang H, Kong X, Mohapatra S, San Juan-Vergara H, Hellermann G, Behera S, Singam R, Lockey RF and Mohapatra SS: Inhibition of respiratory syncytial virus infection with intranasal siRNA nanoparticles targeting the viral NS1 gene. Nat Med 11: 56-62, 2005.

31. Chirivi RG, Garofalo A, Crimmin MJ, Bawden LJ, Stoppacciaro A, Brown PD and Giavazzi R: Inhibition of the metastatic spread and growth of B16-BL6 murine melanoma by a synthetic matrix metalloproteinase inhibitor. Int $\mathrm{J}$ Cancer 58: 460-464, 1994.

32. Kurschat P, Zigrino P, Nischt R, Breitkopf K, Steurer P, Klein CE, Krieg T and Mauch C: Tissue inhibitor of matrix metalloproteinase-2 regulates matrix metalloproteinase- 2 activation by modulation of membrane-type 1 matrix metalloproteinase activity in high and low invasive melanoma cell lines. J Biol Chem 274: 21056-21062, 1999.

33. Vaisanen A, Tuominen H, Kallioinen $M$ and TurpeenniemiHujanen T: Matrix metalloproteinase-2 (72 kD type IV collagenase) expression occurs in the early stage of human melanocytic tumour progression and may have prognostic value. J Pathol 180: 283-289, 1996.
34. Itoh T, Tanioka M, Yoshida H, Yoshioka T, Nishimoto H and Itohara S: Reduced angiogenesis and tumor progression in gelatinase A-deficient mice. Cancer Res 58: 1048-1051, 1998.

35. Vaisanen A, Kallioinen M, Taskinen PJ and TurpeenniemiHujanen T: Prognostic value of MMP-2 immunoreactive protein (72 kD type IV collagenase) in primary skin melanoma. J Pathol 186: 51-58, 1998.

36. Montgomery AM, Mueller BM, Reisfeld RA, Taylor SM and De Clerck YA: Effect of tissue inhibitor of the matrix metalloproteinases- 2 expression on the growth and spontaneous metastasis of a human melanoma cell line. Cancer Res 54: 5467-5473, 1994.

37. Hofmann UB, Eggert AA, Blass K, Brocker EB and Becker JC: Expression of matrix metalloproteinases in the microenvironment of spontaneous and experimental melanoma metastases reflects the requirements for tumor formation. Cancer Res 63: 8221-8225, 2003

38. Hofmann UB, Eggert AA, Blass K, Brocker EB and Becker JC: Stromal cells as the major source for matrix metalloproteinase-2 in cutaneous melanoma. Arch Dermatol Res 297: 154-160, 2005.

39. Li R, Huang L, Guo H and Toole BP: Basigin (murine EMMPRIN) stimulates matrix metalloproteinase production by fibroblasts. J Cell Physiol 186: 371-379, 2001.

40. Sun J and Hemler ME: Regulation of MMP-1 and MMP-2 production through CD147/extracellular matrix metalloproteinase inducer interactions. Cancer Res 61: 2276-2281, 2001.

41. Folkman J: What is the evidence that tumors are angiogenesis dependent? J Natl Cancer Inst 82: 4-6, 1990

42. Fang J, Shing Y, Wiederschain D, Yan L, Butterfield C, Jackson G, Harper J, Tamvakopoulos G and Moses MA: Matrix metalloproteinase- 2 is required for the switch to the angiogenic phenotype in a tumor model. Proc Natl Acad Sci USA 97: 3884-3889, 2000 .

43. Silletti S, Kessler T, Goldberg J, Boger DL and Cheresh DA Disruption of matrix metalloproteinase 2 binding to integrin alpha vbeta 3 by an organic molecule inhibits angiogenesis and tumor growth in vivo. Proc Natl Acad Sci USA 98: 119-124, 2001

44. Kaliski A, Maggiorella L, Cengel KA, Mathe D, Rouffiac V, Opolon P, Lassau N, Bourhis J and Deutsch E: Angiogenesis and tumor growth inhibition by a matrix metalloproteinase inhibitor targeting radiation-induced invasion. Mol Cancer Ther 4: 1717-1728, 2005.

45. Arguello F, Baggs RB and Frantz CN: A murine model of experimental metastasis to bone and bone marrow. Cancer Res 48: 6876-6881, 1988

46. Amundson E, Pradilla G, Brastianos P, Bagley C, Riley LH III, Garonzik IM, McCarthy E, Wolinsky JP and Gokaslan ZL: A novel intravertebral tumor model in rabbits. Neurosurgery 57: 341-346, 2005

47. Thrall JH and Ellis BI: Skeletal metastases. Radiol Clin North Am 25: 1155-1170, 1987.

48. Fornasier VL and Horne JG: Metastases to the vertebral column. Cancer 36: 590-594, 1975. 